\title{
Stability of planetary orbits in binary systems
}

\author{
Z. E. Musielak ${ }^{1,2}$, M. Cuntz ${ }^{2,1}$, E. A. Marshall ${ }^{2}$, and T. D. Stuit ${ }^{3}$ \\ 1 Institut für Theoretische Astrophysik, Universität Heidelberg, Albert Überle Straße 2, 69120 Heidelberg, Germany \\ 2 Department of Physics, University of Texas at Arlington, Arlington, TX 76019-0059, USA \\ e-mail: [zmusielak; cuntz; emarshall]@uta.edu \\ 3 Center for Space Plasma and Aeronomic Research, University of Alabama in Huntsville, Huntsville, AL 35899, USA
}

Received 10 February 2004 / Accepted 23 December 2004

\begin{abstract}
Stability of S-type and P-type planetary orbits in binary systems of different mass and separation ratios is investigated. Criteria for stable, marginally stable and unstable planetary orbits are specified. These criteria are used to determine regions of stability of planetary orbits in different binary systems with Jupiter-type planets. The obtained results show that the regions of stability for S-type orbits depend on the distance ratio between the star and planet, and the stellar companions, in the range of 0.22 and 0.46 , depending on the mass ratio. For P-type orbits, the regions of stability also depend on that distance ratio, in the range of 1.75 and 2.45, again depending on the the mass ratio. Applications of these results to three observed binary systems with giant planets, namely, $\tau$ Boo, HD 195019 and GJ 86, show that the orbits of the giant planets in those systems can be classified as stable, as expected.
\end{abstract}

Key words. stars: binaries: general - celestial mechanics - stars: planetary systems - stars: individual: $\tau$ Boo stars: individual: HD 195019 - stars: individual: GJ 86

\section{Introduction}

The existence of planets around single solar-type stars has been demonstrated by observing the cyclic Doppler shift in photospheric spectral lines. Since this technique favors massive planets with small orbits, all discovered planets are Jupiter-size planets with orbits close to their host stars, although a few planets with sub-Saturn masses have also been recently identified (e.g., Marcy \& Butler 1998, 2000; Marcy et al. 2000; Butler et al. 2002; Marcy et al. 2003; Fischer \& Valenti 2003; McArthur et al. 2004). There has been much theoretical work done in the area of stability of planetary orbits in habitable zones of these newly discovered systems (e.g., Jones et al. 2001; Noble et al. 2002). These studies have shown that terrestrial planets are likely to have stable orbits in many of these systems, largely depending on the location of planetary orbits relative to the stellar habitable zones.

Interestingly, the long-standing question of whether planets can also exist in multiple stellar systems has been answered by observations as arguably at least ten binary and five triple systems with Jupiter-like planets have been identified (e.g., Patience et al. 2002; Eggenberger et al. 2004, and references therein). These systems encompass a broad range of planetary distances and masses, and a very large range of distances of the binary components, with the closest system $20 \mathrm{AU}$ (GJ 86), and the widest system 6400 AU apart (HD 40979). Another binary system hosting a Jupiter-type planet not included in the list by Eggenberger et al. (2004) is HD 168443 (Marcy et al. 2001), in which one of the main components is a brown dwarf. The solar-type star HD 178911B, according Zucker et al. (2002), constitutes the distant component of the system HD $178911 \mathrm{Aa}+\mathrm{b}$ (Tokovinin et al. 2000), indicating that it hosts the first extra-solar planet in a triple system. Zucker et al. also discussed the status of $16 \mathrm{Cyg}$, which is also identified as constituting a triple stellar system with a planet (Lloyd et al. 2002).

The number of known binary or triple stellar systems with planets may actually be larger as some single stars, known to host planets, exhibit a drift in the $\gamma$-velocity, which indicates the presence of a distant companion (Fischer et al. 2001). There is also support for the existence of terrestrial planets in multiple stellar systems based on recent theoretical studies, which show that Earth-mass planets can successfully form in binary (and possibly multiple) stellar systems depending on the stellar masses and separation distances (e.g., Whitmire et al. 1998; Nelson 2000; Kley 2001; Quintana et al. 2002), even though the predominant effect of a companion on the efficiency of planet formation is a negative one. This work, mainly based on ab-initio simulations of planetary accretion, supersedes earlier findings which implied that terrestrial planets would only exist around single stars (e.g., Heppenheimer 1974).

Multiple stellar systems are in fact very common. According to Abt \& Levy (1976), who studied the stellar multiplicity in a sample of 135 F3-G2 IV or V stars, the frequency of these systems is about $65 \%$, with binary systems dominating. This number was revised by Duquennoy \& Mayor (1991) who used a CORAVEL spectroscopic survey. They obtained $43 \%$ 
or $49 \%$ depending on whether a correction for the presence of spurious binaries was applied. In any case, many of these multiple (especially binary) systems are currently observed (or are to be observed in the near future) as the extended search for planets continues. The main goal of this paper is to investigate stability of planetary orbits in binary systems of different mass and separation ratios. The study will help to determine the best candidates for hosting Jupiter-like and Earth-like planets in binary systems.

Planetary orbits in binary systems can be separated into three different categories. Following Dvorak (1986), the first category includes orbits well outside the binary, where the planet essentially orbits the center of mass of both stars (P-type orbit). The second type of orbit is near one of the stars, with the second star to be considered a perturbing agent (S-type orbits). The third type refers to orbits near the triangular Lagrangian points $L_{4}$ or $L_{5}$, which are of little interest here and will not be further discussed, except in conjunction with test simulations (see Sect. 2). An extensive body of literature is devoted to the stability of the $\mathrm{P}$ and S-type orbits in binary systems (e.g., Henon \& Guyot 1970; Szebehely \& McKenzie 1981; Dvorak 1984, 1986; Dvorak et al. 1989; Benest 1988, 1989, 1993, 1996; Kubala et al. 1993; Holman \& Wiegert 1999). Specifically, in the work of Holman and Wiegert, binaries with different eccentricities and mass ratios were considered and regions of stability of both S-type and P-type orbits were identified.

More recently, David et al. (2003) investigated the orbital stability of an Earth-mass planet around a solar-mass star in the presence of a companion star considering a range of companion masses between 0.001 and $0.5 M_{\odot}$. Their work covers a significant portion of the parameter space for S-type orbits studied in this paper, however, they do not consider P-type orbits. David et al. determined the planet's ejection time for systems with different orbital eccentricities and semimajor axes. For fixed companion masses, the ejection time was found to be a steep function of the periastron distance (or orbital radius for circular orbits) to the primary star, a prediction to be compared with the results obtained in this paper.

Further work focused on the study of orbital stability of terrestrial planets in the habitable zone of binary systems. Simulations have been performed for $v$ And, $\gamma$ Cep, GJ 86, and 61 Cyg (Norwood \& Haghighipour 2002; Dvorak et al. 2003; Solovaya \& Pittich 2004; Haghighipour 2005). Models were also given for the systems of $55 \mathrm{Cnc}$ (von Bloh et al. 2003) and GJ 777A (Asghari et al. 2004). In those cases, however, the effect of the binary component on the orbital stability of the terrestrial planet was neglected, owing to the very large separation distances in those systems of 1065 and $3000 \mathrm{AU}$, respectively.

In this paper, we investigate the stability of both $S$ and P-type orbits in binary systems of different mass ratios and separation ratios. All our calculations are performed for circular orbits. The problem of the existence of habitable zones in the considered binary systems is not within the scope of this work and will be addressed in a later paper (Cuntz \& Musielak 2005). Our method and criteria for stable, marginally stable and unstable orbits are described in Sect. 2. Case studies and general results for the stability of the $\mathrm{S}$ and P-type orbits given as functions of mass and separation ratios are presented and discussed in Sect. 3 and Sect. 4, respectively. Applications of our results to specific binary systems that host Jupiter-like planets are described in Sect. 5. Our conclusions are given in Sect. 6.

\section{Method and code testing}

To investigate the stability of $\mathrm{S}$ and P-type orbits in binary systems, we developed a numerical code based on the Runge-Kutta-Fehlberg integration scheme that runs two algorithms simultaneously, i.e., one of fourth-order accuracy and the other of fifth-order accuracy. The difference between each element of the two sets of results are then compared to a user-defined tolerance limit; if any of these differences are larger than the tolerance limit, the step size is reduced and the step is repeated until the differences are acceptable. These differences are approximations of the local truncation error of the fifth-order results of the integration, and provide a measure of error accumulated over the time of simulation. By limiting these accumulated errors to small fractions of their respective elements (position, velocity, and acceleration), the validity of the simulation is guaranteed to fifth-order accuracy. The automatic adjustment of step size is especially useful for orbits that change shape unexpectedly.

In order to validate the code, several tests were performed, namely, two-body and restricted three-body tests. In the two-body test, simulations were performed with a central mass of one solar mass unit (MU) with zero absolute velocity and an orbiting mass of $1 \times 10^{-6} \mathrm{MU}$ at a distance of 2 astronomical units (AU) from the central mass with a velocity of $0.707 \mathrm{AU} / \mathrm{TU}$, where $\mathrm{TU}$ is the time unit that equals $5.0114019 \times 10^{6} \mathrm{~s}$ for a near-circular orbit. The analytical solution for this configuration results in an orbital period of 2.8218 years. The numerical solution after a simulation time of 1600 years resulted in an approximate orbital period of $2.8194 \pm 0.0024$ years, which is within $0.17 \%$ of the analytical solution for the maximal error bar.

In the first restricted three-body case, the following input parameters were considered: central body with $m_{1}=0.75 \mathrm{MU}$, secondary body with $m_{2}=0.25 \mathrm{MU}$ and a body with $m_{3}=$ $1 \times 10^{-3}$ MU. In the first test, the smallest body was placed at either the $\mathrm{L}_{4}$ or $\mathrm{L}_{5}$ Lagrangian stable point and its stability was studied for distances $1 \mathrm{AU}, 2 \mathrm{AU}$ and $5 \mathrm{AU}$ between the central and secondary bodies. Numerical simulations showed that the third body slowly moved away from the stable points after three or more orbits. Decreasing the truncation tolerance from $10^{-10}$ to $10^{-20}$ extended the valid results by approximately $20 \%$. The second restricted three-body test was a straight-line, equal-spacing configuration of three equal masses. The input parameters were chosen such that the masses of all three bodies were $1 \mathrm{MU}$ each, with the central mass having zero velocity and the other two masses on opposite sides separated by $1 \mathrm{AU}$ with velocities of $1 \mathrm{AU} / \mathrm{TU}$ in opposite directions, perpendicular to the line connecting the masses. The comparison between the analytical and numerical results of these two tests provided a good basis for the truncation tolerance selection.

In addition to testing the code against known analytical solutions, we also compared some of our results to those 
obtained by another numerical code that was independently developed and tested by Noble et al. (2002). Good agreement was found between orbits computed by these two different numerical codes.

\section{Initial conditions and stability criteria}

Typical initial conditions for calculating the inner (S-type) and outer (P-type) planetary orbits in binary systems are presented in Fig. 1. The considered configurations are such that the resulting planetary orbits are always circular; this will simplify our consideration of orbital stability in theoretical binary systems. Since the initial positions of both stars and the giant planet are specified, the initial velocity $V_{\mathrm{B}}$ of the secondary star relative to the primary star and the initial velocity $V_{\mathrm{G}}$ of the giant planet relative to the primary star must be determined.

These velocities are computed using the energy equation for circular orbits (e.g., Danby 1988) given as $V_{\text {circ }}=\sqrt{\mu / r}$, where $\mu=G \sum_{i=1}^{N} M_{i}$ with $G$ as universal gravitational constant, $M_{i}$ as masses, and $r$ as orbital radius of the center of mass. In units $\mathrm{MU}, \mathrm{AU}$, and TU, we have $G=1$ and $\mu=M_{\mathrm{A}}+M_{\mathrm{B}}$, with both $M_{\mathrm{A}}$ and $M_{\mathrm{B}}$ given in MU. The velocity $V_{\mathrm{B}}$ is calculated using the above given equation with $\mu=M_{\mathrm{A}}$ and $r=R_{\mathrm{AB}}$, where $R_{\mathrm{AB}}$ is the distance between the primary and secondary star. This two-body system is simulated by the numerical code for a short time to find the initial position and velocity of the center of mass relative to the reference frame fixed to the primary star.

For the inner orbits, the velocity $V_{\mathrm{G}}$ is computed taking $\mu=M_{\mathrm{A}}$, since the primary star is the only body enclosed by the orbit of the planet, and $r=R_{\mathrm{AG}}$, where $R_{\mathrm{AG}}$ is the distance between the primary star and the planet. The result is then added to the velocity of the center of mass of the stars to cancel out the relative motion to produce a near-circular planetary orbit. For the outer orbits, the velocity $V_{\mathrm{G}}$ is again computed using the same equation, but unlike the inner orbit calculation, we have $\mu=M_{\mathrm{A}}+M_{\mathrm{B}}$, since now the planet's orbit encloses both stars. The result is again added to the velocity of the center of mass of the stars to cancel out the relative motion. In addition, the initial position of the planet is adjusted by vectorially adding to it the position of the center of mass of the stars relative to the primary star. This places the initial position of the planet at the same $x$-axis position as the system center-of-mass (see Fig. 1).

These initial conditions were used in all simulations presented in this paper. Of course, initial conditions often have a significant effect on a system that is marginally stable (see below); therefore, our results are by no means absolute in those cases. However, the results showing undeniably stable or unstable orbits are definitely significant in establishing the stability of a given configuration.

The main reason for considering circular orbits is that formal criteria of stability can easily be determined (e.g., Danby 1988). In this paper, orbital stability is determined based on the following criterion: stable (S) $\leq 5 \%$, marginally stable (MS), given as $5 \%<(\mathrm{MS})<35 \%$, and unstable $(\mathrm{U}) \geq 35 \%$, where the percentage refers to the orbital variability with respect to the initial distance between the primary star and the giant planet
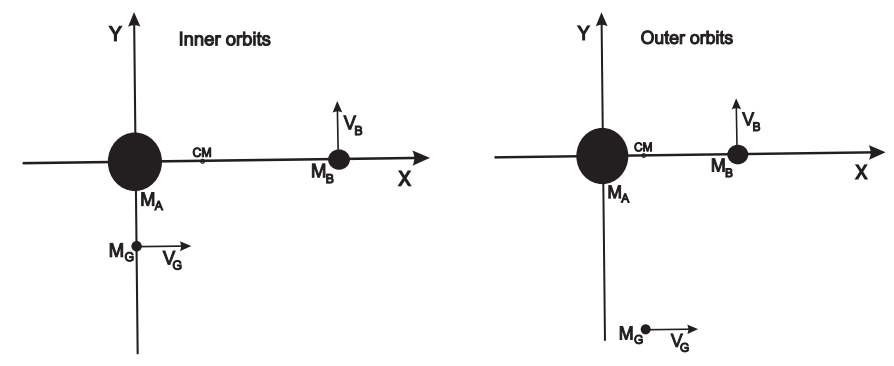

Fig. 1. Initial position of a giant planet with mass $M_{\mathrm{G}}$ relative to the reference frame centered at the primary star with mass $M_{\mathrm{A}}$. The secondary star has mass $M_{\mathrm{B}}$ and the center of mass is denoted by CM. Note that the initial position of the giant planet is different for inner (S-type) and outer (P-type) planetary orbits. The choice of the initial velocities $V_{\mathrm{B}}$ and $V_{\mathrm{G}}$ is described in the main text.

specified at the begin of the calculations (see Fig. 1). The variability is averaged over the number of computed planetary orbits and thereafter the stability criterion is applied. The stability limit of 5\% is motivated by studies of Earth, which seem to indicate that such a stability limit is required for Earth to remain within the conservative habitable zone around the Sun (e.g., Kasting et al. 1993; Underwood et al. 2003), a likely precondition for the evolution of sophisticated forms of life (e.g., Bennett et al. 2003). The limit of 35\% between marginally stable and unstable orbits is based on our own investigations which show that test planets outside that limit are typically not orbitally confined to the respective binary system.

Examples of stable, marginally stable and unstable planetary orbits are shown in Figs. 2-4, respectively. The presented results were obtained for a binary systems with $M_{\mathrm{A}}=1 M_{\odot}$, $M_{\mathrm{B}}=0.6 M_{\odot}, R_{\mathrm{AB}}=8 \mathrm{AU}$ and $M_{\mathrm{G}}=1 M_{\mathrm{Jup}}$. Stable, marginally stable and unstable orbits were calculated by varying the distance between the primary star and the planet, and the following cases were considered: $R_{\mathrm{AG}}=1.60 \mathrm{AU}$ for stable orbits (Fig. 2), $R_{\mathrm{AG}}=2.56 \mathrm{AU}$ for marginally stable orbits (Fig. 3), and finally $R_{\mathrm{AG}}=2.60 \mathrm{AU}$ for unstable orbits (Fig. 4). The results of these simulations are summarized in Table 2. The same stability criteria were applied to both S-type and P-type orbits; for details see Sects. 4.1 and 4.2.

\section{Case studies}

We consider four different theoretical binary systems with the physical properties summarized in Table 1. A Jupiter-type planet $M_{\mathrm{G}}=1 M_{\mathrm{Jup}}$ is included in each system and its distance to the primary star is varied to obtain orbits of different stability. Two of these systems ( 1 and 2 ) have the same masses, but different separations $R_{\mathrm{AB}}$ between the primary and secondary star. The binary system 3 has different masses $M_{\mathrm{A}}$ and $M_{\mathrm{B}}$ as well as different $R_{\mathrm{AB}}$ than the systems 1 and 2 . However, the mass ratio $M_{\mathrm{B}} / M_{\mathrm{A}}$ is the same in all three systems. The binary system 4 differs in both mass and separation ratios from the other systems. The stability of planetary S-type and P-type orbits in these systems is studied using the numerical code described in Sect. 2. Typical timespans for the numerical simulation were 1000 planetary orbital periods. However, in some cases (see below) additional calculations were performed with the 


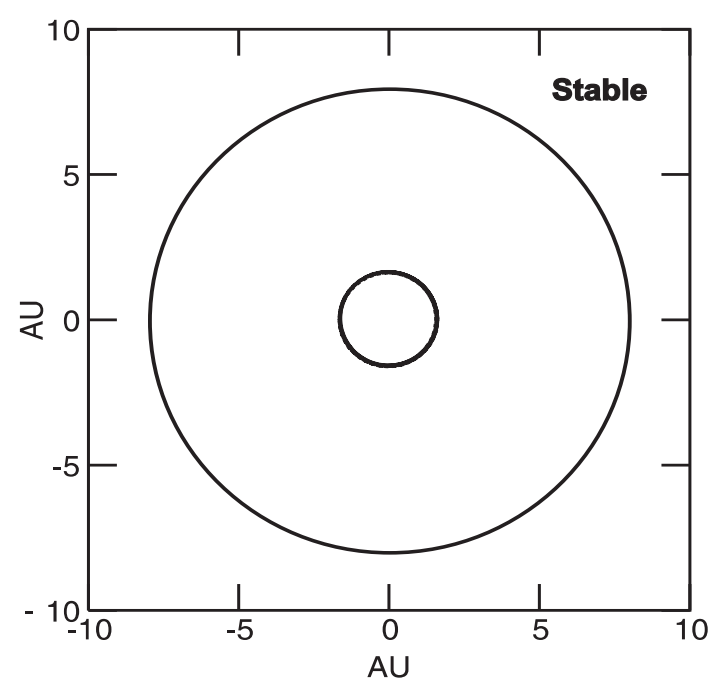

Fig. 2. Stable planetary orbit obtained for the binary system 3 given in Table 1. The criterion for stable orbits is given in the main text.

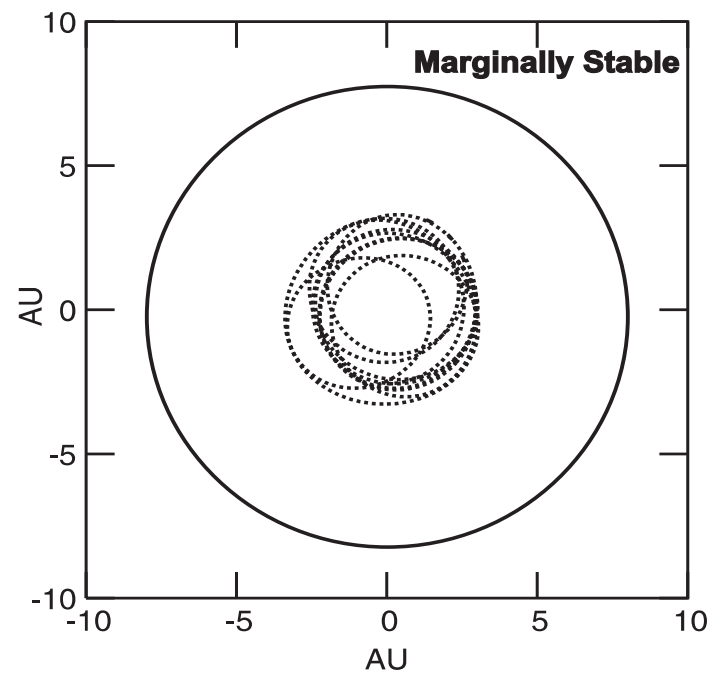

Fig. 3. Marginally stable planetary orbit obtained for the binary system 3 given in Table 1. The criterion for marginally stable orbits is given in the main text.

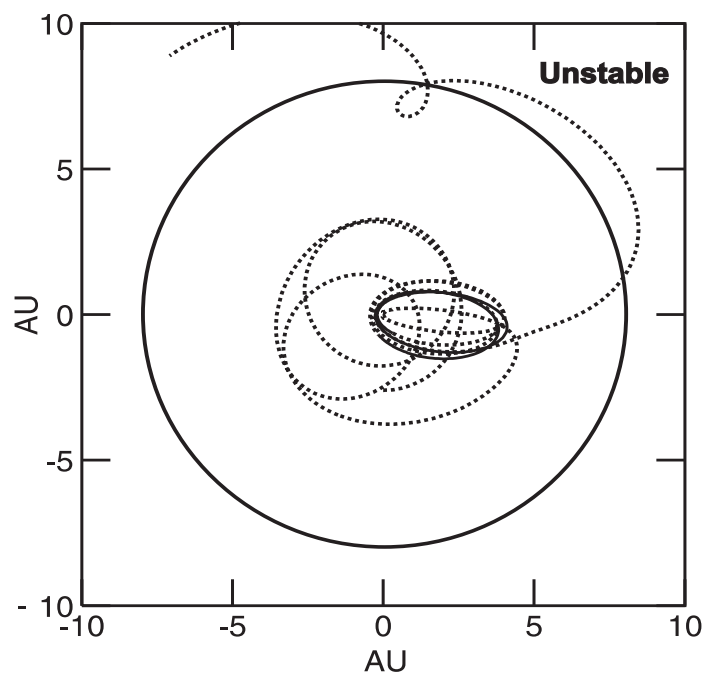

Fig. 4. Unstable planetary orbit obtained for the binary system 3 given in Table 1. The criterion for unstable orbits is given in the main text.
Table 1. Theoretical binary systems.

\begin{tabular}{ccccc}
\hline \hline $\begin{array}{c}\text { Binary } \\
\text { system }\end{array}$ & $\begin{array}{c}M_{\mathrm{A}} \\
{\left[M_{\odot}\right]}\end{array}$ & $\begin{array}{c}M_{\mathrm{B}} \\
{\left[M_{\odot}\right]}\end{array}$ & $M_{\mathrm{B}} / M_{\mathrm{A}}$ & $\begin{array}{c}R_{\mathrm{AB}} \\
{[\mathrm{AU}]}\end{array}$ \\
\hline 1 & 1.25 & 0.75 & 0.60 & 15 \\
2 & 1.25 & 0.75 & 0.60 & 10 \\
3 & 1.00 & 0.60 & 0.60 & 8 \\
4 & 0.75 & 0.25 & 0.33 & 5 \\
\hline
\end{tabular}

Table 2. Stability of S-type orbits in binary systems.

\begin{tabular}{cccc}
\hline \hline $\begin{array}{c}\text { Binary } \\
\text { system }\end{array}$ & $\begin{array}{c}R_{\mathrm{AG}} \\
{[\mathrm{AU}]}\end{array}$ & $R_{\mathrm{AG}} / R_{\mathrm{AB}}$ & $\begin{array}{c}\text { Orbital } \\
\text { stability }\end{array}$ \\
\hline 1 & 3.000 & 0.200 & $\mathrm{~S}$ \\
& 4.000 & 0.275 & $\mathrm{MS}$ \\
& 4.750 & 0.320 & $\mathrm{MS}$ \\
& 4.850 & 0.325 & $\mathrm{U}$ \\
\hline 2 & 2.000 & 0.200 & $\mathrm{~S}$ \\
& 2.750 & 0.275 & $\mathrm{MS}$ \\
& 3.200 & 0.320 & $\mathrm{MS}$ \\
& 3.250 & 0.325 & $\mathrm{U}$ \\
\hline 3 & 1.600 & 0.200 & $\mathrm{~S}$ \\
& 2.200 & 0.275 & $\mathrm{MS}$ \\
& 2.560 & 0.320 & $\mathrm{MS}$ \\
& 2.600 & 0.325 & $\mathrm{U}$ \\
\hline 4 & 1.000 & 0.200 & $\mathrm{~S}$ \\
& 1.375 & 0.275 & $\mathrm{~S}$ \\
& 1.600 & 0.320 & $\mathrm{MS}$ \\
& 1.625 & 0.325 & $\mathrm{MS}$ \\
& 2.000 & 0.400 & $\mathrm{U}$ \\
\hline
\end{tabular}

Note: Simulations were performed for giant planets with $M_{\mathrm{G}}=1 M_{\text {Jup }}$ based on 1000 orbits.

simulation time increased to 150000 and 40000 orbital periods for S-type and P-type orbits, respectively. In our calculations of observed binary systems, we used up to $10^{5}$ orbital periods (see Sect. 5).

\subsection{Stability of S-type orbits}

Various different cases have been studied and the most important results obtained for inner (S-type) orbits are summarized in Tables 2 and 3. According to Table 2, orbital stability is the same for the binary systems 1,2 and 3, which have the same mass ratios $M_{\mathrm{B}} / M_{\mathrm{A}}=0.6$, but different masses $M_{\mathrm{A}}$, $M_{\mathrm{B}}$ and separation distances $R_{\mathrm{AB}}$. Different separation ratios of $R_{\mathrm{AG}} / R_{\mathrm{AB}}$ between 0.200 and 0.325 result in stable, marginally stable and unstable planetary orbits, respectively. However, the orbital stability behavior becomes different when the mass ratio is changed and the separation ratio remains the same compared to the previous systems (see binary system 4 of Table 2). This can be shown even more clearly by taking the 
Table 3. Dependence of orbital stability on mass of secondary star.

\begin{tabular}{ccccc}
\hline $\begin{array}{c}\text { Binary } \\
\text { system }\end{array}$ & $M_{\mathrm{B}} / M_{\mathrm{A}}$ & $\begin{array}{c}R_{\mathrm{AG}} \\
{[\mathrm{AU}]}\end{array}$ & $R_{\mathrm{AG}} / R_{\mathrm{AB}}$ & $\begin{array}{c}\text { Orbital } \\
\text { stability }\end{array}$ \\
\hline 3 & 0.20 & 2.56 & 0.32 & $\mathrm{~S}$ \\
& 0.40 & 2.56 & 0.32 & $\mathrm{MS}$ \\
& 0.60 & 2.56 & 0.32 & $\mathrm{MS}$ \\
& 0.80 & 2.56 & 0.32 & $\mathrm{U}$ \\
\hline
\end{tabular}

Note: Simulations were performed for giant planets with $M_{\mathrm{G}}=1 M_{\text {Jup }}$ based on 1000 orbits.

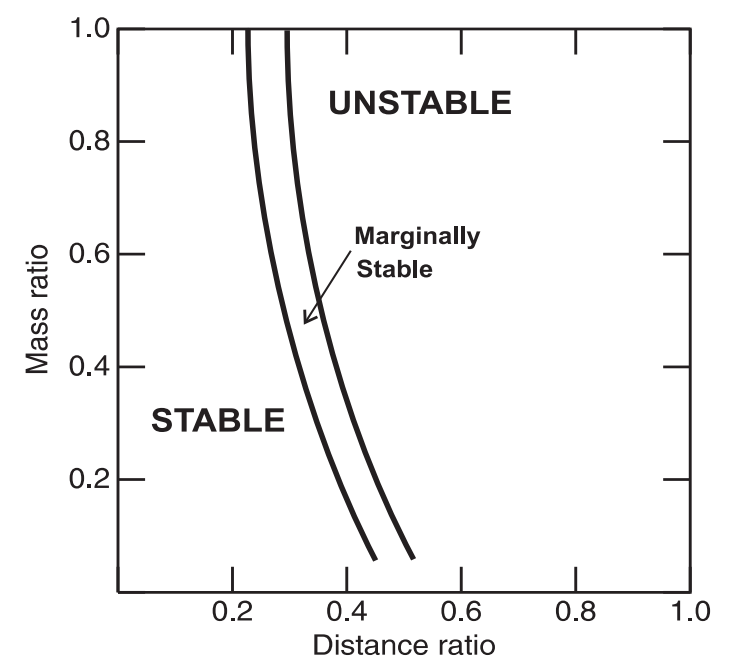

Fig. 5. Range of mass ratios and separation ratios corresponding to stable, marginally stable and unstable inner (S-type) planetary orbits in binary systems. The simulations are based on 1000 orbits.

separation ratio to be constant and changing the mass ratio by varying the mass of the secondary star (see Table 3 ). Hereby we assumed $M_{\mathrm{A}}=1 M_{\odot}$ and $R_{\mathrm{AB}}=8 \mathrm{AU}$. Now the orbits change from stable to unstable when the mass ratio is increased from 0.2 to 0.8 . These results suggest that the regions of stability of planetary orbits in binary systems are determined both by the mass and separation ratio.

The next step is to compute the boundaries separating planetary orbits of different stability for binary systems of different mass and separation ratios. These boundaries are plotted in Fig. 5, which shows that the inner orbits can be divided into two distinct classes, namely, stable and unstable (given by the two solid curves in Fig. 5). According to our stability criteria (see Sect. 3), orbits in the region between the two curves can be classified as marginally stable. It is interesting that the region of stable orbits decreases with increasing mass ratio. In addition, the region of marginally stable orbits reaches its maximum for the lowest mass ratio of 0.05 , indicative that the classification is sensitive to the considered stability criteria (see Figs. 2 to 4 ) and the adopted initial conditions (see Fig. 1). The boundary separating stable orbits from marginally stable orbits changes from a distance ratio of 0.22 for a stellar mass ratio of 1.0 to 0.46 for a mass ratio of 0.05 .

Furthermore, we tested the stability of the simulations over more extended runs, by inspecting the degree of orbital stability for mass ratio/distance ratio points just inside the domain of orbital stability. We found that for 150000 planetary orbits corresponding to 0.6 to $1.4 \times 10^{6}$ years, depending on the system, the range of orbital variability stays between 5.11 to $6.02 \%$ compared to 5.12 to $6.00 \%$ for the baseline models of 1000 planetary orbits (see Table 4). This approach allows us to highlight the time development, if any, of small variations. The results obtained for mass ratios $M_{\mathrm{B}} / M_{\mathrm{A}}$ between 0.1 and 0.9 seem to indicate that the domain of stability is essentially independent on the length of the model run pursued, at least if the time of simulation exceeds 1000 planetary orbits.

These results reconfirm that regions of stability for S-type orbits exist in binary systems, in agreement with previous results. For example, Benest (1993, 1996) showed that nearly circular orbits on the order of a quarter of the periastron separation between two stars are stable in the $\eta$ Coronae Borealis, $\alpha$ Centauri and Sirius systems. The mass ratios of these systems are in the range of 0.45 to 0.66 , which is consistent with stable orbits presented in Fig. 5. Further comparisons with the more recent work by David et al. (2003) will be given in Sect. 4.3. The latter authors investigated a similar range of companion masses, but assumed an Earth-mass rather than a Jupiter-mass planet for their orbital stability analyses.

The location of the boundaries shown in Fig. 5 also depends on $M_{\mathrm{G}}$, to a certain extent. To assess this dependency, we increased $M_{\mathrm{G}}$ to $5 M_{\mathrm{Jup}}$ and computed the resulting shift of the boundaries. Now the region of stability of each S-type system is increased on the order of $10 \%$ (i.e., between 8.5 and $13.6 \%$ ). In addition, the boundary between the stable and marginally stable zone is shifted toward larger distance ratios, but only on the order of $2 \%$. Consequently, the region of stable S-type orbits in Fig. 5 becomes larger when the mass of the giant planet $M_{\mathrm{G}}$ is increased while the masses $M_{\mathrm{A}}$ and $M_{\mathrm{B}}$ remain unchanged.

\subsection{Stability of P-type orbits}

We also investigated the stability of outer (P-type) orbits for all binary systems given in Table 1 . The main conclusion from these studies is similar to that obtained for the inner (S-type) orbits, namely, that the region of stability of P-type orbits is determined by the mass and separation ratios. Selected results are given in Table 5 . The orbits can be classified as either stable, marginally stable, or unstable. Our simulations show that all outer orbits classified as marginally stable after the simulation time of 10 planetary orbital periods became unstable after the time of simulations has been extended (see Table 6). Interestingly, test simulation for binary system 2 showed that the point in time for this transition critically depends on the distance $R_{\mathrm{AG}}$. For $R_{\mathrm{AG}}=22.325 \mathrm{AU}$, this transition occurred after 20 orbital periods, whereas for $R_{\mathrm{AG}}=22.5 \mathrm{AU}$, the transition happened after $10^{5}$ orbital periods. For some distances $R_{\mathrm{AG}}$, even orbits originally classified as stable became unstable. Nevertheless, we always found a distance $R_{\mathrm{AG}}$, for which the orbit remains stable even after long simulation times such as $4 \times 10^{4}$ orbital periods.

Figure 6 shows the boundaries between stable, marginally stable, and unstable P-type orbits. Selected results are given in 
Table 4. Time development of orbital variability near stability boundary for S-type orbits.

\begin{tabular}{cccccccc}
\hline \hline$M_{\mathrm{B}} / M_{\mathrm{A}}$ & $R_{\mathrm{B}} / R_{\mathrm{A}}$ & $\begin{array}{c}1 \mathrm{~K} \\
{[\%]}\end{array}$ & $\begin{array}{c}10 \mathrm{~K} \\
{[\%]}\end{array}$ & $\begin{array}{c}50 \mathrm{~K} \\
{[\%]}\end{array}$ & $\begin{array}{c}75 \mathrm{~K} \\
{[\%]}\end{array}$ & $\begin{array}{c}100 \mathrm{~K} \\
{[\%]}\end{array}$ & $\begin{array}{c}150 \mathrm{~K} \\
{[\%]}\end{array}$ \\
\hline 0.10 & 0.44 & 5.11929 & 5.10191 & 5.10581 & 5.10811 & 5.10752 & 5.106775 \\
0.15 & 0.39 & 5.21648 & 5.21112 & 5.21076 & 5.20963 & 5.20931 & 5.210078 \\
0.33 & 0.32 & 5.19342 & 5.19629 & 5.19107 & 5.19522 & 5.19489 & 5.195642 \\
0.50 & 0.28 & 5.13434 & 5.11435 & 5.12381 & 5.12558 & 5.11732 & 5.117495 \\
0.60 & 0.27 & 5.63816 & 5.62302 & 5.63053 & 5.62834 & 5.62294 & 5.625883 \\
0.90 & 0.24 & 5.99959 & 6.03042 & 6.01998 & 6.02526 & 6.02137 & 6.015876 \\
\hline
\end{tabular}

Note: Simulations were performed for giant planets with $M_{\mathrm{G}}=1 M_{\mathrm{Jup}}$.

Table 5. Stability of P-type orbits in binary systems.

\begin{tabular}{ccccc}
\hline \hline $\begin{array}{c}\text { Binary } \\
\text { system }\end{array}$ & $M_{\mathrm{B}} / M_{\mathrm{A}}$ & $\begin{array}{c}R_{\mathrm{AG}} \\
{[\mathrm{AU}]}\end{array}$ & $R_{\mathrm{AG}} / R_{\mathrm{AB}}$ & $\begin{array}{c}\text { Orbital } \\
\text { stability }\end{array}$ \\
\hline 2 & 0.60 & 22.0 & 2.20 & $\mathrm{U}$ \\
& & 23.0 & 2.30 & $\mathrm{MS}$ \\
& & 24.0 & 2.40 & $\mathrm{~S}$ \\
\hline 4 & 0.33 & 11.0 & 2.20 & $\mathrm{U}$ \\
& & 11.1 & 2.22 & $\mathrm{MS}$ \\
& & 11.2 & 2.24 & $\mathrm{~S}$ \\
\hline
\end{tabular}

Note: Simulations were performed for giant planets with $M_{\mathrm{G}}=1 M_{\mathrm{Jup}}$ based on 1000 orbits.

Table 6. Dependence of orbital stability on simulation time.

\begin{tabular}{cccc}
\hline \hline $\begin{array}{c}\text { Binary } \\
\text { system }\end{array}$ & $\begin{array}{c}R_{\mathrm{AG}} \\
{[\mathrm{AU}]}\end{array}$ & $\begin{array}{c}\text { Orbital } \\
\text { periods }\end{array}$ & $\begin{array}{c}\text { Orbital } \\
\text { stability }\end{array}$ \\
\hline 2 & 22.325 & 10 & $\mathrm{MS}$ \\
& 22.325 & 20 & $\mathrm{U}$ \\
\hline & 22.350 & 10 & $\mathrm{MS}$ \\
& 22.350 & 20 & $\mathrm{MS}$ \\
& 22.350 & 30 & $\mathrm{MS}$ \\
& 22.350 & 40 & $\mathrm{MS}$ \\
& 22.350 & 50 & $\mathrm{U}$ \\
\hline & 22.500 & $10^{1}$ & $\mathrm{MS}$ \\
& 22.500 & $10^{2}$ & $\mathrm{MS}$ \\
& 22.500 & $10^{3}$ & $\mathrm{MS}$ \\
& 22.500 & $10^{4}$ & $\mathrm{MS}$ \\
& 22.500 & $10^{5}$ & $\mathrm{U}$ \\
\hline
\end{tabular}

Note: Simulations were performed for giant planets with $M_{\mathrm{G}}=1 M_{\mathrm{Jup}}$.

Table 6. The results depicted in Fig. 6 demonstrate that the minimum radius of stable circular orbits increases from a distance ratio of 1.75 to 2.45 , when the mass ratio is increased (due to increasing $M_{\mathrm{B}}$ ), although it remains about constant for mass ratios larger than 0.4 . The existence of this "critical" mass ratio of approximately 0.4 is an interesting result as it shows that changes in the sphere of influence of a binary system resulting

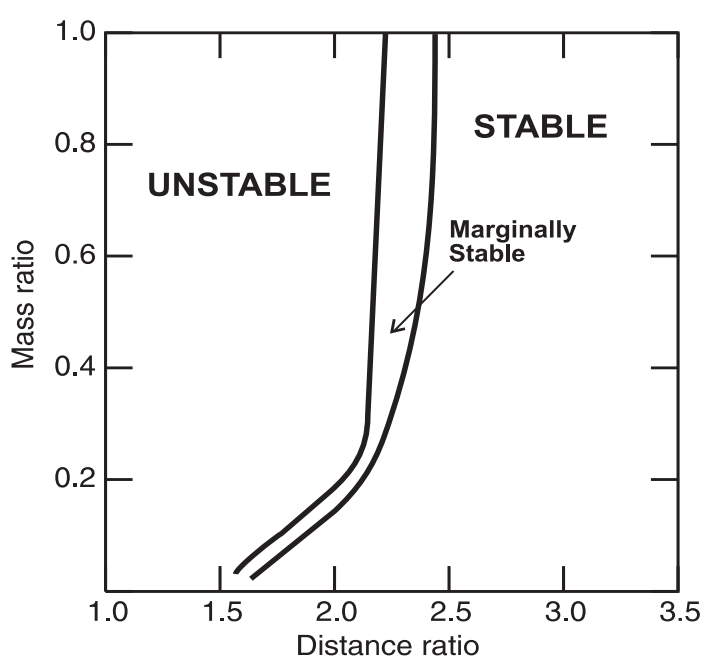

Fig. 6. Range of mass ratios and sparation ratios corresponding to stable, marginally stable and unstable outer (P-type) planetary orbits in binary systems. The simulations are based on 1000 orbits.

from the increasing $M_{\mathrm{B}}$ do not influence orbital stability of a giant planet as long as the mass ratio is beyond that value. The comparison between Figs. 5 and 6 also shows that this result is only valid for the P-type orbits.

We again explored the stability of the simulations over a more extended time frame. Therefore, we chose time periods of 30000 or 40000 planetary orbits corresponding to 2.7 to $3.3 \times 10^{6}$ years, depending on the system, compared to the baseline of 1000 orbits. The distance points were again chosen just inside the domain of stable orbits. We found that the orbital variability stays between 4.04 to $4.99 \%$ compared to 4.16 to $5.00 \%$ for the baseline models of 1000 planetary orbits (see Table 7). These results again confirm that the domains of stability are essentially independent of the lengths of the simulationat least beyond a minimal time of simulation.

The results shown in Fig. 6 have counterparts in Kubala et al. (1993). Here, two plots can be used for comparison - a plot of Hill's critical orbital radii and a plot combining the critical radii of Graziani \& Black (1981) using a Laplace method and a Hill's method approach (e.g., Danby 1988). It should be noted that Kubala et al. (1993) used a different definition of mass ratio than used here. They defined $\mu=\left(M_{\mathrm{B}}+M_{\mathrm{G}}\right) / 2 M_{\mathrm{A}}$ as the mass ratio. The plot of Hill's critical orbit radii shows a 
Table 7. Time development of orbital variability near stability boundary for P-type orbits.

\begin{tabular}{cccccccc}
\hline \hline$M_{\mathrm{B}} / M_{\mathrm{A}}$ & $R_{\mathrm{B}} / R_{\mathrm{A}}$ & $\begin{array}{c}1 \mathrm{~K} \\
{[\%]}\end{array}$ & $\begin{array}{c}5 \mathrm{~K} \\
{[\%]}\end{array}$ & $\begin{array}{c}10 \mathrm{~K} \\
{[\%]}\end{array}$ & $\begin{array}{c}20 \mathrm{~K} \\
{[\%]}\end{array}$ & $\begin{array}{c}30 \mathrm{~K} \\
{[\%]}\end{array}$ & $\begin{array}{c}40 \mathrm{~K} \\
{[\%]}\end{array}$ \\
\hline 0.10 & 1.95 & 4.16005 & 4.11282 & 4.08047 & 4.05153 & 4.03336 & 4.04495 \\
0.15 & 2.05 & 4.19949 & 4.19578 & 4.19493 & 4.19248 & 4.19492 & 4.19569 \\
0.33 & 2.25 & 4.81418 & 4.70511 & 4.79329 & 4.79473 & 4.79914 & $\ldots$ \\
0.50 & 2.35 & 4.99639 & 4.99501 & 4.99456 & 4.99473 & 4.99439 & $\ldots$ \\
0.60 & 2.40 & 4.98571 & 4.98039 & 4.98185 & 4.98188 & 4.98139 & $\ldots$ \\
0.90 & 2.45 & 4.99734 & 4.99471 & 4.99501 & 4.99483 & 4.98991 & $\ldots$ \\
\hline
\end{tabular}

Note: Simulations were performed for giant planets with $M_{\mathrm{G}}=1 M_{\text {Jup }}$.

peak at a radius of about 2.4 at approximately $\mu=0.4$ (which corresponds to a mass ratio $M_{\mathrm{B}} / M_{\mathrm{A}} \simeq 0.8$ ). The combination plot, in which the lower critical radius is used so that both stability criteria are satisfied, peaks at a radius of about 2.4 at $\mu=$ 0.25 (which corresponds to a mass ratio $M_{\mathrm{B}} / M_{\mathrm{A}} \simeq 0.5$ ). The curve representing the boundary between stable and marginally stable orbits in Fig. 6 peaks at a radius just near 2.45 at a mass ratio of about 0.4 in agreement with the results described here.

Dvorak et al. (1989) also considered P-type planetary orbits. They compared their results with the results from two other papers, Henon \& Guyot (1970) and Szebehely \& McKenzie (1981) in tabular form for mass ratios of 0.1 to 0.5 , defined in the same way as by Kubala et al. (1993). While these authors defined only a single stability boundary, Dvorak et al. (1989) defined an upper and lower critical orbit. Their lower critical orbit ranges from 1.7 at a 0.1 mass ratio to 1.9 at a 0.5 mass ratio. The upper critical orbit ranges from 2.15 at 0.1 to 2.3 at 0.5 mass ratio, with a peak of 2.4 at a mass ratio of 0.2 . Henon \& Guyot showed almost no variation with mass ratio and the critical orbit radius is about 2.14; the general shape of the curves presented by these authors is very similar to our results shown in Fig. 6. Szebehely \& McKenzie's critical orbits ranges from 2.27 at a mass ratio of 0.1 , to a peak of 2.45 at 0.25 mass ratio, to 2.17 at 0.5 mass ratio. All these data are in the vicinity of the results of this paper, even though the peaks appear at lower mass ratios. Dvorak et al. (1989) concluded that the stability regions are independent of the mass ratio. The results presented in this paper show that there is indeed a range of mass ratios for which this conclusion is correct.

\subsection{Remarks on orbital stability}

The results of this investigation indicate that domains of stability exist for planetary orbits in various theoretical stellar binary systems and that these domains can be generalized to any binary system based on the mass and distance ratios of the various components involved - a result in agreement with previous investigations. In fact, only the mass ratio is relevant for defining the zones of stability, not the individual masses of the stars themselves, which is a key point in the characterization of the dynamics of stellar binary systems. The boundary curves for stable orbits in Figs. 5 and 6 define limits for the periods of stable planetary orbits in binary systems. The leftmost curve in
Table 8. Comparison with results by David et al.

\begin{tabular}{cccc}
\hline \hline$M_{\mathrm{B}} / M_{\mathrm{A}}$ & $\begin{array}{c}\text { Boundary S/MS } \\
{[\mathrm{AU}]}\end{array}$ & $\begin{array}{c}\text { Boundary MS/U } \\
{[\mathrm{AU}]}\end{array}$ & $\begin{array}{c}\text { DQFA } \\
{[\mathrm{AU}]}\end{array}$ \\
\hline 0.1 & $0.437 \pm 0.001$ & $0.457 \pm 0.001$ & $0.31 \pm 0.04$ \\
0.5 & $0.278 \pm 0.001$ & $0.348 \pm 0.001$ & $0.28 \pm 0.02$ \\
\hline
\end{tabular}

Note: Simulations were performed for giant planets with $M_{\mathrm{G}}=1 M_{\mathrm{Jup}}$, whereas David et al. (2003; DQFA) assumed Earth-mass planets.

Fig. 5 (S-type orbits) corresponds to maximal separation ratios between 0.22 and 0.46 , depending on the mass ratio of the stellar components, whereas the rightmost curve in Fig. 6 (P-type orbits) corresponds to minimal separation ratios between 1.75 and 2.45, again given by the stellar mass ratio. This information can be incorporated into Kepler's third law to obtain a relation for a conservative maximum (minimum) period for the stable inner (outer) orbits of planets in binary systems.

These findings can be compared to other results in the literature, particularly the work by David et al. (2003). They studied the orbital stability of an Earth-mass planet around a solar-mass star in the presence of a companion star for a range of companion masses between 0.001 and $0.5 M_{\odot}$. In fact, they considered both circular and elliptical orbits, and derived expressions for the expected ejection time of the planet from the system. For fixed companion masses, the ejection time was found to be a steep function of the periastron distance (or orbital radius for circular orbits) to the primary star. David et al. predict that the domain of orbital stability gets progressively smaller over time according to the logarithm of the time of simulation. Table 4 depicting the possible time evolution of planetary instability for S-type orbits, however, does not indicate this type of behavior, as the domain of stability does not change noticeably beyond 1000 planetary orbits (at least not within the time frame of up to 150000 planetary orbits). Nonetheless, we were able to estimate the consistency of the boundary between stable and marginally stable orbits based on the formula for the planetary ejection times by David et al. (2003) for simulations of up to $10^{6}$ years. We found full consistency for the mass ratio $M_{\mathrm{B}} / M_{\mathrm{A}}=0.5$, but no consistency for the lower mass ratio of $M_{\mathrm{B}} / M_{\mathrm{A}}=0.1$ (see Table 8 ). A possible reason for this disagreement might be that David et al. considered Earth-mass planets rather than Jupiter-mass planets in their simulations, 
Table 9. Stability of S-type orbits in binary systems with observed giant planets.

\begin{tabular}{lcccccccc}
\hline \hline $\begin{array}{l}\text { Binary } \\
\text { system }\end{array}$ & $\begin{array}{c}M_{\mathrm{A}} \\
{\left[M_{\odot}\right]}\end{array}$ & $\begin{array}{c}M_{\mathrm{B}} \\
{\left[M_{\odot}\right]}\end{array}$ & $M_{\mathrm{B}} / M_{\mathrm{A}}$ & $\begin{array}{c}M_{\mathrm{G}} \\
{\left[M_{\mathrm{Jup}}\right]}\end{array}$ & $\begin{array}{c}R_{\mathrm{AB}} \\
{[\mathrm{AU}]}\end{array}$ & $\begin{array}{c}R_{\mathrm{AG}} \\
{[\mathrm{AU}]}\end{array}$ & $R_{\mathrm{AG}} / R_{\mathrm{AB}}$ & $\begin{array}{c}\text { Orbital } \\
\text { stability }\end{array}$ \\
\hline$\tau$ Boo & 1.3 & 0.4 & 0.3 & 3.87 & 225 & 0.0462 & $2.1 \times 10^{-4}$ & $\mathrm{~S}$ \\
HD 195019 & 1.0 & 0.7 & 0.7 & 3.51 & 157 & 0.14 & $8.9 \times 10^{-4}$ & $\mathrm{~S}$ \\
GJ 86 & 0.8 & 0.05 & 0.06 & 4.00 & 20 & 0.11 & $5.5 \times 10^{-3}$ & $\mathrm{~S}$ \\
\hline
\end{tabular}

which may have an increased impact for models involving a companion star with a relatively low mass.

Finally, we should also comment on the uncertainty bar assigned to the planetary ejection times deduced in the simulations by David et al. (2003). Here it should be noted that these authors studied a large variety of models involving planets at different angular starting positions, whereas in our work both the companion star and the giant planet were placed at the 3 o'clock position in all simulations. David et al. found that the ejection critically depends on those starting positions in the cases of orbital instability - a result in full agreement with our previous work (Noble et al. 2002). A significantly reduced uncertainty bar is implied if the model simulations would have been restricted to a distinct starting position as in the present work.

\section{Binary systems with observed giant planets}

\subsection{Observations}

Here we explore the orbital stability of planets in realistic binary systems. As stated in Sect. 1, there are at least 10 binary systems with known Jupiter-type planets (Patience et al. 2002; Eggenberger et al. 2004, and references therein). In all these systems, the Jupiter-type planets are located on inner (S-type) orbits as so far no systems with giant planets located on outer (P-type) orbits have been found. In this work, we consider only cases in which the planet is orbiting its host star in a circular or quasi-circular orbit. Therefore, the permissible planetary eccentricity is assumed to be $e \leq 0.05$. This condition limits the number of target systems to three, namely, $\tau$ Boo, HD 195019, and GJ 86. Their physical properties are briefly described below and summarized in Table 9.

$\tau$ Boo $(\mathrm{F} 7 \mathrm{~V})$ is a well-studied star with a very massive Jupiter-type planet with a minimum mass of $3.87 M_{\text {Jup }}$. The orbital distance of the planet is $0.0462 \mathrm{AU}$ (Butler et al. 1997), allowing the planet to be classified as one of 20 or so closein giant planets discovered to date. Incidentally, the planet has also been suspected to increase the activity level of its host star, as first pointed out by Cuntz et al. (2000) and recently verified observationally by Shkolnik et al. (2003). The orbital eccentricity of the planet is given as $e=0.018 \pm 0.016$ (Butler et al. 1997). Patience et al. (2002) pointed out that $\tau$ Boo constitutes an eccentric binary system with $\tau$ Boo $\mathrm{A} \simeq 1.3 M_{\odot}, \tau$ Boo $\mathrm{B}$ $\simeq 0.4 M_{\odot}$ and a semi-major axis of $225 \mathrm{AU}$. Even though our method so far has only been proposed for systems with circular orbits for both the planets and the binary components, we included $\tau$ Boo as a general illustration.
Table 10. Time development of orbital variability in binary systems with observed giant planets.

\begin{tabular}{lcccc}
\hline \hline $\begin{array}{l}\text { Binary } \\
\text { system }\end{array}$ & $1 \mathrm{~K}$ & $10 \mathrm{~K}$ & $100 \mathrm{~K}$ & $150 \mathrm{~K}$ \\
{$[\%]$} & {$[\%]$} & {$[\%]$} & {$[\%]$} \\
\hline$\tau$ Boo & 0.283994 & 0.284011 & 0.284353 & 0.283526 \\
HD 195019 & 0.334530 & 0.334614 & 0.335292 & 0.335452 \\
GJ 86 & 0.469386 & 0.469395 & 0.470148 & 0.471522 \\
\hline
\end{tabular}

HD 195019 is a G3 IV-V star that is orbited by a Jupiter-type planet. Based on the analysis of Doppler measurements obtained at Lick Observatory, Fischer et al. (1999) identified Keplerian velocity variations with a period of 18.27 days, an orbital eccentricity of $e=0.03 \pm 0.03$, indicative of a minimum planet mass of $3.51 M_{\text {Jup }}$. The semi-major axis of the orbit was determined as $a=0.14$ AU. HD 195019 was later identified as a member of a wide binary system with a separation distance of 157 AU (Allen et al. 2000). The secondary star is a mid-type $\mathrm{K}$ dwarf star with a likely mass of about $0.7 M_{\odot}$ (Gray 1992). The mass of the primary star was estimated as $0.98 \pm 0.06 M_{\odot}$ based on its spectral type (Allen et al. 2000).

GJ 86 (=HD 13445) is an example of a system where a brown dwarf constitutes a companion to a star that is orbited by a planet. Queloz et al. (2000) reported a $4 M_{\text {Jup }}$ planet (minimum mass) with a 15.8 day orbital period and a $0.11 \mathrm{AU}$ semi-major axis around GJ 86A, a K dwarf star. The inferred eccentricity is $e=0.046 \pm 0.004$. The planet detection has been made through very precise radial velocity measurements with the CORALIE echelle spectrograph. Els et al. (2001) concluded that GJ 86A has an additional companion GJ 86B in an orbit with a semi-major axis somewhat larger than $20 \mathrm{AU}$, also previously suggested by Queloz et al. (2000). GJ 86B has been identified to be at the transition from $\mathrm{L}$ to $\mathrm{T}$ dwarfs with a mass of 40-70 $M_{\text {Jup }}$ based on atmospheric temperature models (Burrows et al. 1997). Here we assume $50 M_{\text {Jup }}$ for simplicity.

\subsection{Orbital stability of the observed systems}

The results regarding the orbital stability of the planets in our three target systems are given in Tables 9 and 10. It is found that in all three systems, the planets are identified as stable (S), as expected. The mass ratios $M_{\mathrm{B}} / M_{\mathrm{A}}$ for the stellar components in the systems $\tau$ Boo, HD 195019, and GJ 86 are given as $0.3,0.7$, and 0.06 , respectively. Furthermore, the ratios of the separation distances between the stellar components and the stars and planets $R_{\mathrm{AG}} / R_{\mathrm{AB}}$ (see Sect. 4.1) are $2.1 \times 10^{-4}$, 
$8.9 \times 10^{-4}$, and $5.5 \times 10^{-3}$, respectively. Therefore, in all these cases, the Jupiter-type planets are well within the stable parameter domain, and are far away from the stable/marginally stable separation line (see Fig. 5). In fact, the range of orbital variability, inspected in simulations of up to 150000 planetary orbits, remains close to $0.28 \%$ for $\tau$ Boo, $0.34 \%$ for HD 195019 , and $0.47 \%$ for GJ 86 (see Table 10). Thus, the fact that in reality those planetary orbits are slightly elliptical does not appear to be relevant here as no significant modifications are expected to occur.

\section{Conclusions}

We investigated the stability of S and P-type planetary orbits in binary systems of different mass and separation ratios using a numerical code based on a Runge-Kutta-Fehlberg integration scheme. The integration scheme is based on the simultaneous computation of two algorithms, i.e., one of fourth-order and one of fifth-order accuracy, allowing the choice of the integration time step to attain optimal accuracy. Base line calculations are pursued for 1000 planetary orbits to distinguish different cases of stability, but a large number of runs extend to more than $10^{5}$ planetary orbits.

In the study of S-type and P-type planetary orbits, we specified criteria for stable, marginally stable and unstable circular planetary orbits. We found that the separation lines between the different stability regimes depend both on the mass and separation ratios of the stellar system components. For S-type planetary orbits, stable orbits are obtained for distance ratios between 0.22 and 0.46 , depending on the mass ratio. For P-type planetary orbits, stable orbits are obtained for distance ratios between 1.75 and 2.45, again depending on the the mass ratio. The results were compared to those previously obtained, including work by Holman \& Wiegert (1999) and David et al. (2003), and we found our results largely consistent with those earlier findings.

An interesting result was found for P-type orbits. Our results demonstrate that the minimum radius of stable circular orbits remains practically constant for all mass ratios larger than 0.4. The existence of this "critical" mass ratio clearly shows that changes in the sphere of influence of a binary system resulting from increasing the companion star mass $M_{\mathrm{B}}$ do not influence orbital stability of a giant planet as long as the mass ratio remains beyond that value.

Finally, our studies of the stability of planetary orbits in three observed binary systems with giant planets, namely, $\tau$ Boo, HD 195019 and GJ 86, were based on simulations extending up to 150000 planetary orbits. They showed that the orbits of the giant planets in those systems remained well within the stable domain during the time of simulations, as expected.

Acknowledgements. This work has been supported by the Alexander von Humboldt Foundation (Z.E.M.) and the University of Texas at Arlington through its Research Enhancement Program (M.C.).

\section{References}

Abt, H. A., \& Levy, S. G. 1976, ApJS, 30, 273

Allen, C., Poveda, A., \& Herrera, M. A. 2000, A\&A, 356, 529

Asghari, N., Broeg, C., Carone, L., et al. 2004, A\&A, 426, 353

Benest, D. 1988, Celest. Mech. Dyn. Astron., 43, 47

Benest, D. 1989, A\&A, 223, 361

Benest, D. 1993, Celest. Mech. Dyn. Astron., 56, 45

Benest, D. 1996, A\&A, 314, 983

Bennett, J., Shostak, S., \& Jakosky, B. 2003, Life in the Universe (San Francisco: Addison Wesley)

Burrows, A., Marley, M., Hubbard, W. B., et al. 1997, ApJ, 491, 856

Butler, R. P., Marcy, G. W., Fischer, D. A., et al. 2002, in Planetary Systems in the Universe: Observation, Formation and Evolution, ed. A. Penny, P. Artymowicz, A.-M. Lagrange, \& S. Russell (San Francisco: ASP), IAU Symp., 202, in press

Butler, R. P., Marcy, G. W., Williams, E., Hauser, H., \& Shirts, P. 1997, ApJ, 474, L115

Cochran, W. D., Hatzes, A. P., Butler, R. P., \& Marcy, G. W. 1997, ApJ, 483, 457

Cuntz, M., Saar, S. H., \& Musielak, Z. E. 2000, ApJ, 533, L151

Cuntz, M., \& Musielak, Z. E. 2005, Icarus, in preparation

Danby, J. M. A. 1988, Fundamentals of Celestial Mechanics (Richmond: Willmann-Bell, Inc.)

David, E.-M., Quintana, E. V., Fatuzzo, M., \& Adams, F. C. 2003, PASP, 115,825

Duquennoy, A., \& Mayor, M. 1991, A\&A, 248, 485

Dvorak, R. 1984, Celest. Mech. Dyn. Astron., 34, 369

Dvorak, R. 1986, A\&A, 167, 379

Dvorak, R., Froeschle, Ch., \& Froeschle, Cl. 1989, A\&A, 226, 335

Dvorak, R., Pilat-Lohinger, E., Funk, B., \& Freistetter, F. 2003, A\&A, 398, L1

Eggenberger, A., Udry, S., \& Mayor, M. 2004, A\&A, 417, 353

Els, S. G., Sterzik, M. F., Marchis, F., et al. 2001, A\&A, 370, L1

Fischer, D. A., Marcy, G. W., Butler, R. P., Vogt, S. S., \& Apps, K. 1999, PASP, 111, 50

Fischer, D. A., Marcy, G. W., Butler, R. P., et al. 2001, ApJ, 551, 1107

Fischer, D. A., \& Valenti, J. A. 2003, in Scientific Frontiers in Research on Extrasolar Planets, ed. D. Deming, \& S. Seager, ASP Conf. Ser., 294, 117

Gray, D. F. 1992, The Observation and Analysis of Stellar Photospheres, Cambridge Astrophys. Ser., 20 (Cambridge: Cambridge Univ. Press)

Graziani, F., \& Black, D. C. 1981, ApJ, 251, 337

Haghighipour, N. 2005, ApJ, submitted

Henon, M., \& Guyot, M. 1970, Periodic Orbits, Stability and Resonances, ed. G. E. O. Giacaglia (Dordrecht: Reidel), 349

Heppenheimer, T. A. 1974, Icarus, 22, 436

Holman, M. J., \& Wiegert, P. A. 1999, AJ, 117, 621

Jones, B. W., Sleep, P. N., \& Chambers, J. E. 2001, A\&A, 366, 254

Kasting, J. F., Whitmire, D. P., \& Reynolds, R. T. 1993, Icarus, 101, 108

Kley, W. 2001, in The Formation of Binary Stars, ed. H. Zinnecker, \& R. D. Mathieu (San Francisco: ASP), IAU Symp., 200, 511

Kubala, A., Black, D., \& Szebehely, V. G. 1993, Celest. Mech. Dyn. Astron., 56, 51

Lloyd, J. P., et al. 2002, in Planetary Systems in the Universe: Observation, Formation and Evolution, ed. A. Penny, P. Artymowicz, A.-M. Lagrange, \& S. Russell (San Francisco: ASP), IAU Symp., 202, in press

Marcy, G. W., \& Butler, R. P. 1998, ARA\&A, 36, 57

Marcy, G. W., \& Butler, R. P. 2000, PASP, 112, 137

Marcy, G. W., Cochran, W. D., \& Mayor, M. 2000, in Protostars and Planets IV, ed. V. Mannings, A. P. Boss, \& S. S. Russell (Tucson: Univ. of Arizona Press), 1285 
Marcy, G. W., Butler, R. P., Fischer, D. A., \& Vogt, S. S. 2003, in Scientific Frontiers in Research on Extrasolar Planets, ed. D. Deming, \& S. Seager, ASP Conf. Ser., 294, 1

Marcy, G. W., Butler, R. P., Vogt, S. S., et al. 2001, ApJ, 555, 418

McArthur, B. E., Endl, M., Cochran, W. D., et al. 2004, ApJ, 614, L81

Nelson, A. F. 2000, ApJ, 537, L65

Noble, M., Musielak, Z. E., \& Cuntz, M. 2002, ApJ, 572, 1024

Norwood, J., \& Haghighipour, N. 2002, BAAS, 34, 892

Patience, J., White, R. J., Ghez, A. M., et al. 2002, ApJ, 581, 654

Queloz, D., Mayor, M., Weber, L., et al. 2000, A\&A, 354, 99

Quintana, E. V., Lissauer, J. J., Chambers, J. E., \& Duncan, M. J. 2002, ApJ, 576, 982

Shkolnik, E., Walker, G. A. H., \& Bohlender, D. A. 2003, ApJ, 597, 1092 (Erratum: 2004, ApJ, 609, 1197)
Solovaya, N. A., \& Pittich, E. M. 2004, Contributions of the Astronomical Observatory Skalnaté Pleso, 34, 105

Szebehely, V. G., \& McKenzie, R. 1981, Celest. Mech. Dyn. Astron., 23,3

Tokovinin, A. A., Griffin, R. F., Balega, Yu. Yu., Pluzhnik, E. A., \& Udry, S. 2000, Astron. Let., 26, 116 (translated from Astr. Zh., 26, 146)

Underwood, D. R., Jones, B. W., \& Sleep, P. N. 2003, Int. J. Astrobiology, 2, 289

von Bloh, W., Cuntz, M., Franck, S., \& Bounama, C. 2003, Astrobiology, 3, 681

Whitmire, D. P., Matese, J. J., Criswell, L., \& Mikkola, S. 1998, Icarus, 132, 196

Zucker, S., Naef, D., Latham, D. W., et al. 2002, ApJ, 568, 363 